\title{
Optical twisters: beams having twists in both phase and amplitude
}

\author{
Vincent Ricardo Daria*a ${ }^{\mathrm{a}}$, Darwin Palima ${ }^{\mathrm{b}}$ and Jesper Glückstad*b \\ ${ }^{a}$ ARC Centre for Quantum Atom Optics, Australian National University, Australia \\ ${ }^{\mathrm{b}}$ DTU Fotonik, Dept. of Photonics Engineering, Technical University of Denmark, Denmark \\ *Corresponding authors: vincent.daria@anu.edu.au and jesper.gluckstad@fotonik.dtu.dk
}

\begin{abstract}
We describe a diffracting beam with orbital angular momentum (OAM) but with a helical profile in both phase and amplitude components of the beam. This is different from Laguerre-Gaussian (LG) beams where only the phase component has a helical profile. Such profile in LG beams introduces a phase singularity at the centre and produces a dark region surrounded by a ring-shaped light pattern. For LG-beams, the ring radius is proportional to the degree of helicity or topological charge of the beam. The beam we describe here is initially characterized with an apodized helical phase front at the outskirts and linearly scaled towards no phase singularity at the centre of the beam. At the focal volume, we show that our beam forms an intensity distribution that can be accurately described as an "optical twister" as it propagates in the forward direction. Unlike LG beams, an optical twister can have minimal changes in radius but with a scalable OAM. Furthermore, we characterize the OAM in terms of its capacity to introduce spiral motion on particles trapped along its orbit. We also show that our "optical twister" maintains a high concentration of photons at the focus even as the topological charge is increased. Such beams can be applied to fundamental studies of light and atoms such as in quantum entanglement of the OAM, toroidal traps for cold atoms and for optical manipulation of microscopic particles.
\end{abstract}

Keywords: Optical trapping and manipulation, orbital angular momentum, helico-conical beams

\section{INTRODUCTION}

The orbital angular momentum (OAM) [1,2] of optical fields with phase singularities have been instrumental in creating super-fluid vortex modes in Bose-Einstein condensates [3-5], rotating cold atoms [6] and revolving microscopic particles [7-11]. Optical fields with OAM are commonly associated with Laguerre-Gaussian (LG) beams and optical vortices, which are characterized with a helical phase expressed by $\exp (-i l \varphi)$, where $l$ is the topological charge that describes the degree of the helical phase profile. In quantum theory, the topological charge relates to a quantized orbital angular momentum of $l \hbar$ per photon [1-2], and has been used to demonstrate experiments on the entanglement of photons [12,13]. However, unlike the limited options in handedness of spin angular momentum of a photon, which is associated with the polarization of light, the OAM of a photon is proportional to the topological charge and can be theoretically increased indefinitely.

An optical vortex or light beam with helical phase introduces a component of the Poynting vector along the azimuthal angle. When such a beam undergoes strong focusing, the azimuthal components destructively interfere giving rise to a dark centre surrounded by a high intensity ring of light via constructive interference. As the topological charge is increased, the area where destructive interference at the centre increases thereby enlarging the light ring. The propagation along the optical axis follows a conical ray of light where the concentration of high intensities is maintained at the outskirts of the conical beam where constructive interference is takes place. In a quantum mechanical perspective, one can perceive that the probability of detecting photons with OAM is optimal within the conical ring of light and negligible at the central dark region.

Increasing the OAM disperses the distribution of photons around a larger ring. This affects experiments in cold atoms, quantum entanglement and micromanipulation that strongly depend on the transfer or detection of OAM of photons. The limiting factor depends on the balance to maintain high concentration of photons with a large OAM. 
Here, we describe a diffracting beam with a spiral profile on both the amplitude and phase of the beam. The spiral beam is a special case of a general set of Helico-Conical beams described in our previous work [14]. This family of beams is initially characterized with an apodized helical phase front at the outskirts and linearly scaled towards no phase singularity at the center of the beam. At the focus, we show that diffracting spiral beams have minimal changes in radius but with a scalable OAM. Further, we characterize an increasing OAM in terms of its capacity to introduce spiral motion on particles trapped along its orbit and show that spiral beams maintain a high concentration of photons at the focus even as the topological charge is increased.

\section{3D STRUCTURE OF DIFFRACTING SPIRAL BEAMS}

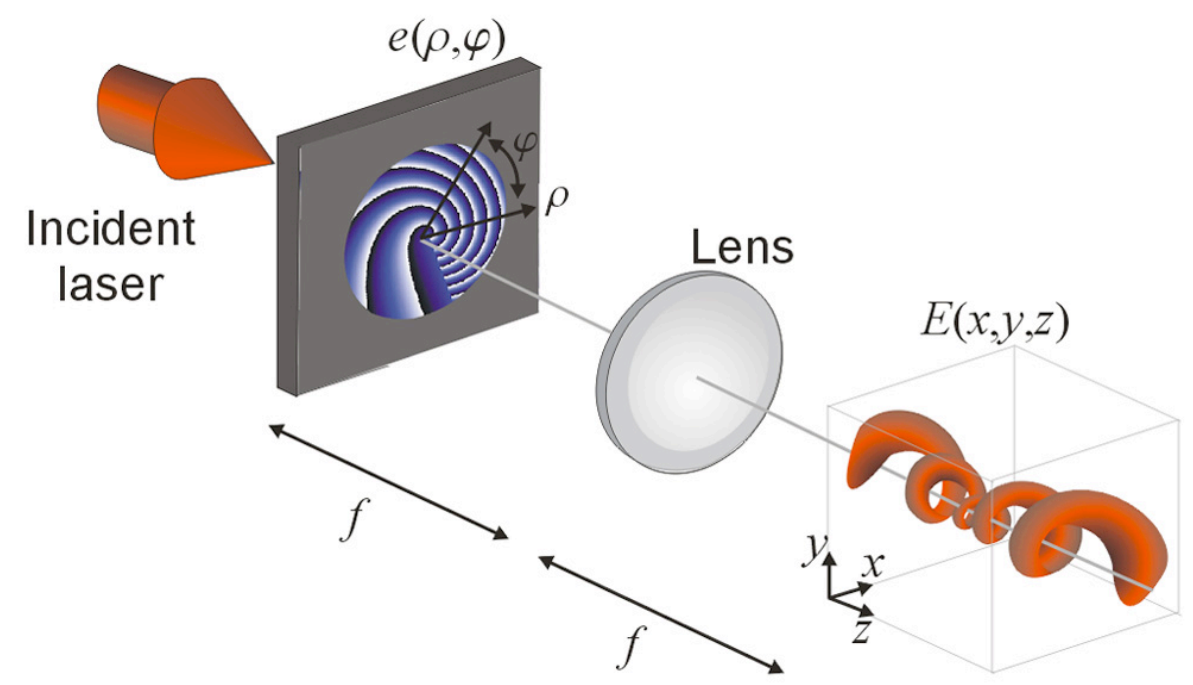

Figure 1. Conceptual overview of the setup for generating optical twisters.

The spiral beams we use here is a subset of Helico-Conical beams introduced and described in our earlier work [14]. Figure 1 shows a conceptual overview of the system. The beam is produced via holographic micro-projection method $[8,15,16]$ where the field of an incident laser is encoded with an appropriate phase pattern at the back focal plane of a Fourier transforming lens. The input field is given by

$$
e(\rho, \varphi)=A(\rho) \exp \left[-i\left(l \varphi \frac{\rho}{\rho_{o}}\right)\right]
$$

where $l$ is the topological charge, $\rho$ is the radial distance from the optical axis, $\varphi$ is the azimuthal angle and $A(\rho)$ is a circular aperture of radius $\rho_{\mathrm{o}}$. After the lens, the beam tapers towards a smallest radius at the focus and enlarges after the focus. Hence, the beam has a region of highest concentration of photons at the focus. We have previously described the properties of the phase profile in Eq. 1 as a non-separable multiplication between a Laguerre-Gaussian (LG) mode of charge $l$ and a conical phase of unitary scale. The incorporation of a conical phase varies the helical phase linearly generating a distinctly different beam as compared to LG and Bessel beams.

The three-dimensional (3D) intensity distribution after the lens transformation, $I\left(k_{r}, k_{s}, k_{z}\right)$, is difficult to derive analytically due to the fact that the phase at the input has an inseparable dependence between $\rho$ and $\varphi$. To visualize the intensity distribution at the output, we numerically evaluate the intensity distribution via the Fresnel diffraction integral [17] which incorporates a lens function of numerical aperture, $\mathrm{NA}=0.2$. To compare with a standard focusing without any phase encoding, we set $l=0$ (see fig 2 .a and 2.b) to yield an intensity distribution that can be characterized as a typical Airy function for a specified NA and incident laser wavelength. Figure 2.a shows the 3D projection visualized via ImageJ (ver 1.40g National Institutes of Health, USA). It is derived from 150 planes of intensity distributions calculated via the Fresnel diffraction integral. The inset shows the intensity distribution as viewed from the top. The color map at the right describes the density of photons. Also for comparison, we visualize $3 \mathrm{D}$ projections of the intensity distribution 
of LG "doughnut" beams for topological charges $l=10$ (fig 2.b) and $l=20$ (fig 2.c). LG beams are produced by setting $\rho=\rho_{\mathrm{o}}$ in the phase term of Eq. 1. As the topological charge is increased, light in an LG beam has lesser photon density since it is distributed to a ring with a larger radius.

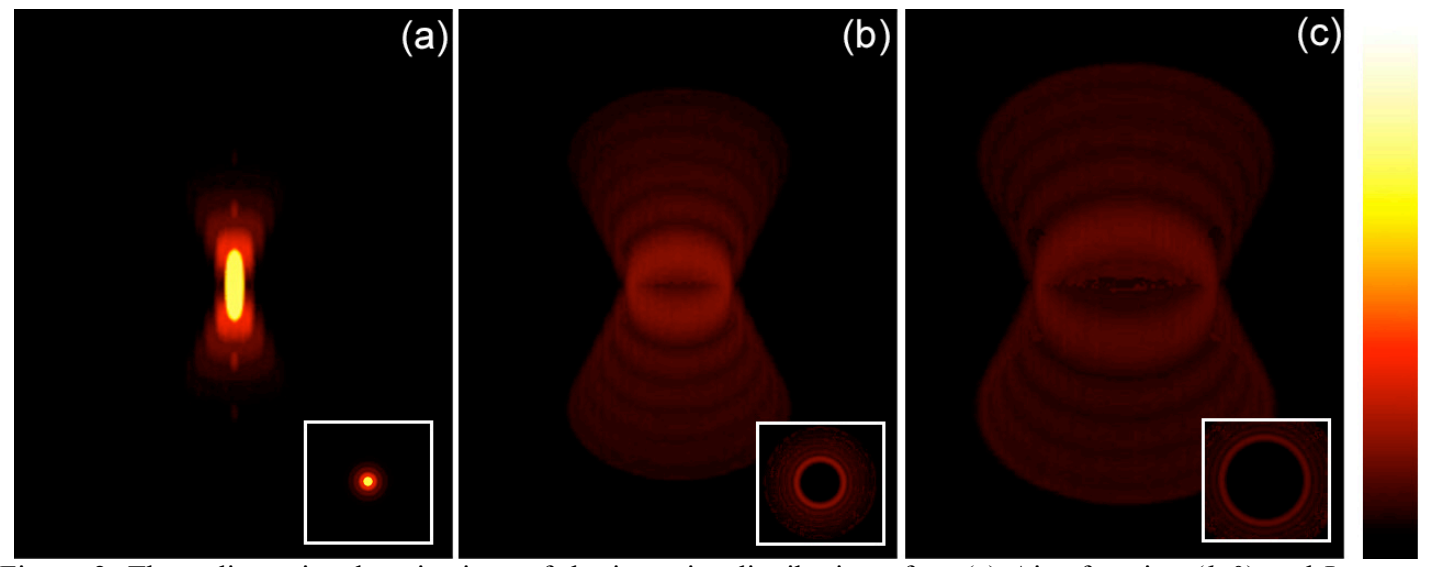

Figure 2. Three-dimensional projections of the intensity distribution of an (a) Airy function $(l=0)$ and Laguerre Gaussian beams with topological charge (b) $l=10$ and (c) $l=20$. The color map at the right represents decreasing photon density from top to bottom.

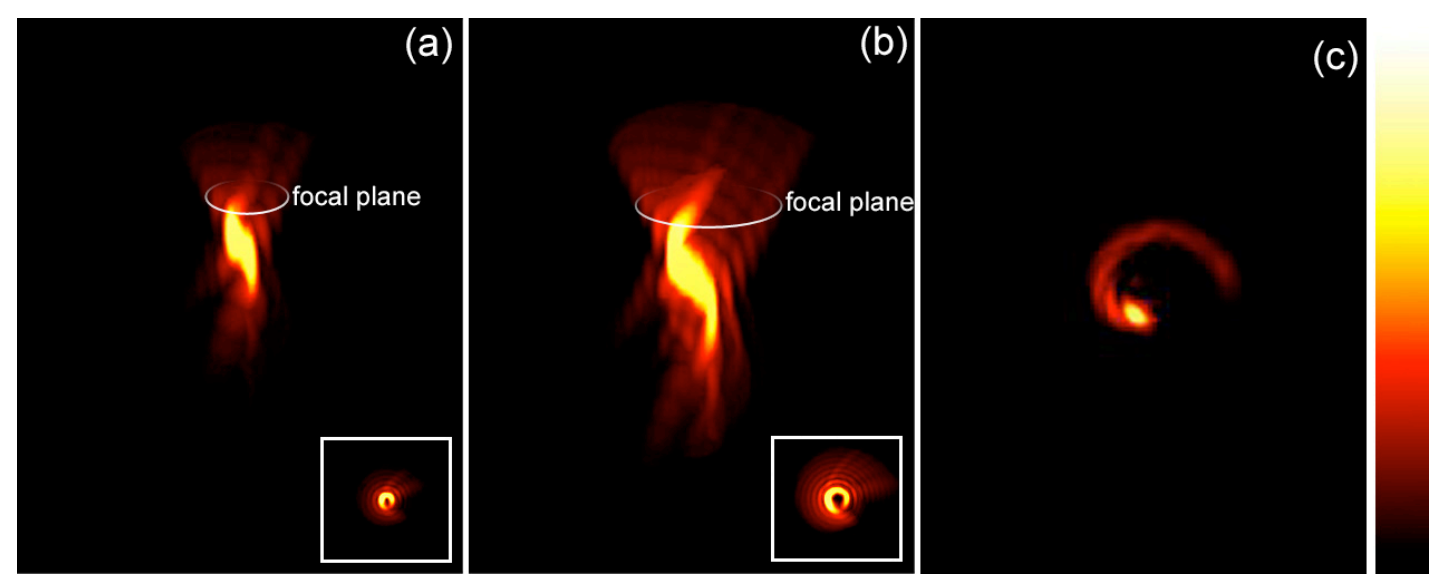

Figure 3. Three-dimensional projections of the intensity distribution of optical beams having both spiral profile in both amplitude and phase distribution for (a) $l=10$ and (b) $l=20$. For a positive $l$, the beam twists around a clockwise direction as the beam propagates. The focus is also shifted from the nominal focus. (c) The intensity distribution at the focal plane for $l=20$. The color map at the right represents decreasing photon density from top to bottom.

The 3D projections of the beams described in this work are shown in Fig. 3. The beam's region of highest photon density spirals and tapers towards a focal point, which is shifted as compared to the nominal focus of the lens. Fig. 3.a shows the optical twister for $l=10$. The inset in fig. 3.a shows the top view with the highest density of photons concentrated near the focal point. Compared to an LG beam of similar charge, the radius at the focal point of the optical twister is significantly smaller. The photon density is also significantly higher for the optical twister. Fig. 3.b shows an optical twister for $l=20$, which shows a more pronounced twist in the intensity distribution. Fig. 3.c shows the intensity distribution at the nominal focal plane of the lens, which is slightly higher than the focal point of the optical twister. The intensity distribution illustrates an asymmetric curved beam with one end closer to the optical axis, while the other end is dispersed outwards towards an increasing radius. A negative topological charge will shift the focal point of the optical twister before the nominal focus of the lens. The beam will also spiral in the opposite direction. 
We can determine the maximum intensity from the numerical beam propagation results. We can also track the radial position of the maximum intensity. Figure 4.a shows the maximum intensities ( $I_{\mathrm{LG}}$ and $\left.I_{\mathrm{S}}\right)$ as a function of topological charge for LG and spiral beams, respectively. As the topological charge is increased, we expect a decrease in intensity due to diffracted light to higher orders. Figure 4.b shows the radial position of highest intensity for both LG and spiral beams. The rate at which the radius of LG beams increases with topological charge is $6 \mathrm{X}$ higher than spiral beams. Such indicates that majority of the beams are concentrated within a small region around the optical axis.

While the output power is a good measure of optical throughput, it does not account how the photons are concentrated along the ring of a doughnut beam and the spiral beams described here. The power is normally derived by integrating the intensity over the area for a specified plane, $z$, such that $d P=I\left(k_{\rho}, k \varphi\right) k_{\rho} d k_{\rho} d k_{\varphi}$, where $k_{\varphi}$ and $k_{\varphi}$ are spatial frequency polar coordinates after the lens and $I\left(k_{\rho}, k_{\varphi}\right)$ is the intensity distribution at the plane, $k_{z}$, giving the highest photon density. To characterize the two-dimensional photon concentration around the ring, we calculate $d P / d k_{\rho}=I\left(k_{\rho}, k \varphi\right) k_{\rho} \int d k_{\varphi}$. For LG beams, we assume a full ring where we integrate from 0 to $2 \pi$. The intensity distribution for LG beams with topological charge, $l$, is also constant along the ring such that $d P / d k_{\rho}=I_{L G}(l) 2 \pi k k_{\rho}$. For spiral beams, however, the intensity distribution along a plane only covers a fraction of the full azimuthal angle such that $d P / d k_{\rho}=I_{S}(l) C \pi k_{\rho}$, where $C$ is a constant fraction of the full angle, which can be approximately set to $3 / 2$. Figure 4.c shows the plot $d P / d k_{\rho}$, which characterizes the two-dimensional photon concentration for both LG and spiral beams as a function of $l$. With the assumption of a constant $C$, the absolute value for photon concentrations for both LG and spiral beams are quantitatively incomparable. However, we can qualitatively show that the photon concentration for spiral beams is maintained with increasing $l$. The rate at which the radius of spiral beams increase is slow such that it maintains the photon concentration despite the fact that such beams experience losses to higher diffraction orders as $l$ is increased. On the other hand, LG beams drastically decrease in photon concentration as a function of $l$ following a high rate of increase in ring radius on top of losses to high diffraction orders.
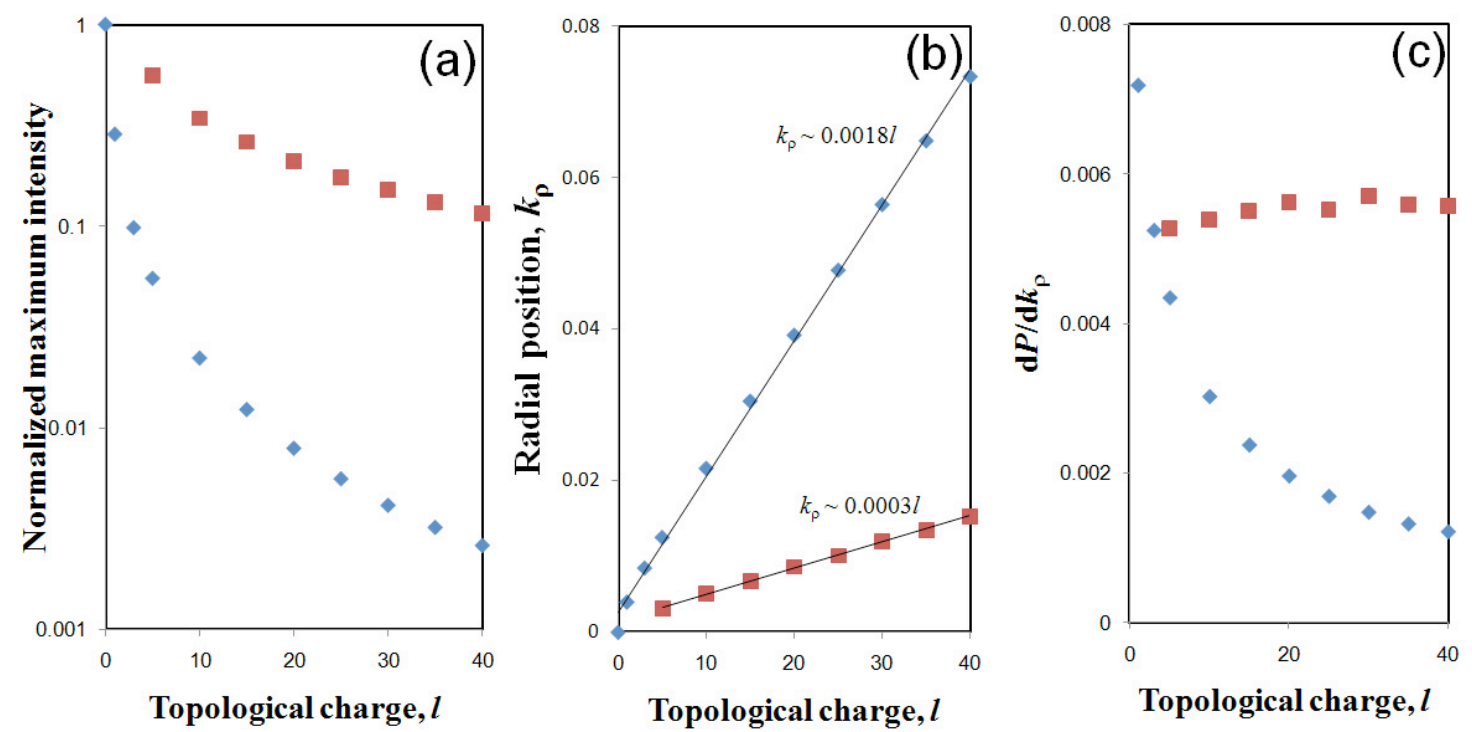

Figure 4. Characterization plots for spiral ( $\square$ ) and LG beams ( $>$ ) showing: (a) Normalized maximum intensity; (b) radius of the ring, $k_{p}$; and (c) two-dimensional photon concentration, $\mathrm{d} P / \mathrm{d} k_{\mathrm{p}}$ as a function of topological charge, $l$.

\section{EXPERIMENT}

The optical setup is shown in figure 5, which is similar to a standard SLM-based optical tweezers setup where diffractively generated light patterns are demagnified and relayed to a sample through an inverted microscope [7, 15, 
16]. This setup is used to analyze the spiral profile of optical twisters and its capacity to transfer both linear and orbital angular momentum on microscopic particles. We used a collimated laser source from a continuous wave (CW) near infrared (NIR) Ti:S laser (Spectra Physics 3900s) pumped by a Nd:YV04 laser (Spectra Physics Millenia). The NIR laser beam $(\lambda \sim 800 \mathrm{~nm})$ is expanded (via L1 and L2) to illuminate the full area of the spatial light modulator (SLM, Hamamatsu Photonics). The second lens (L2) of the folded beam expander also functions as an optical Fourier transform lens for the phase-encoded reflection from the SLM to form spiral beams at the Fourier plane. Reusing lens L2 minimizes the tilt angle between the incident and phase encoded beam. Minimizing the tilt produces high degree of purity when encoding LG and twister beams. The beams are translated into micron scale at the sample volume using L3 and objective lens arranged in a $4 f$ lens configuration. The dichroic mirror is chosen to reflect the incident NIR and transmit visible light, which carries image information of the sample.

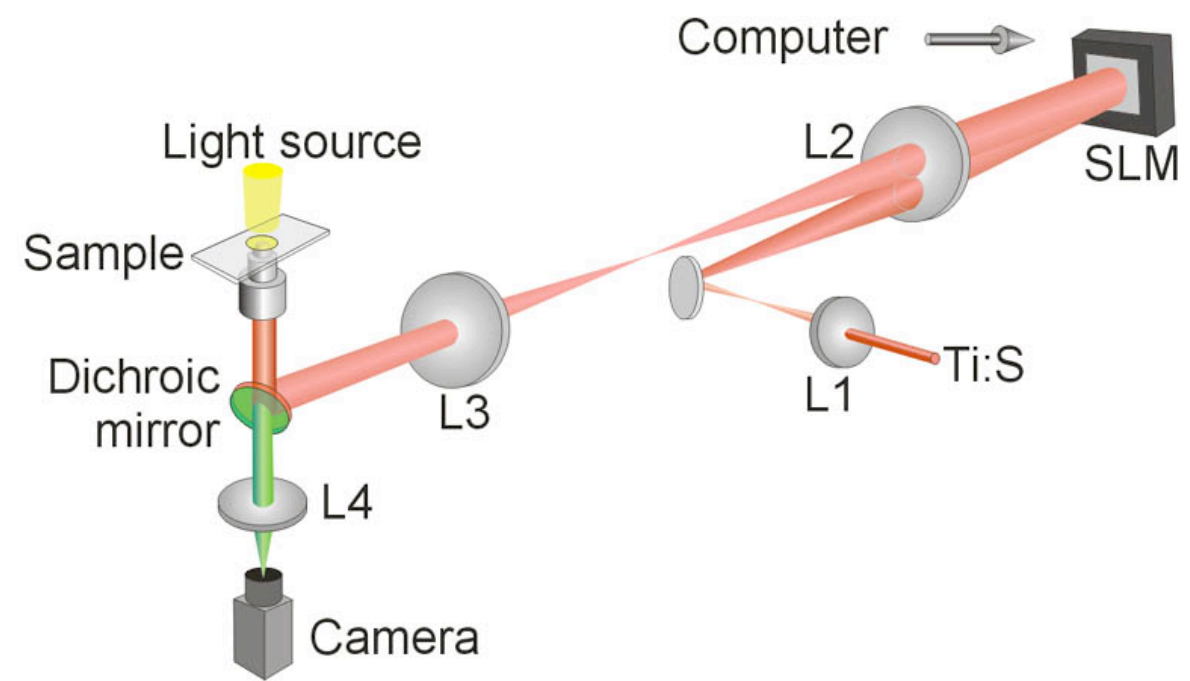

Figure 5. Optical setup for analyzing the spiral characteristics of an optical twister and its capacity to transfer linear and orbital angular momentum on microscopic dielectric particles.

The two-dimensional phase pattern is derived using the phase of the function given in equation 1. Encoding of the hologram on the SLM is achieved via a secondary video output with $800 \times 600$ pixel resolution. A program developed in Labview (National Instruments) enables control of the SLM to encode phase patterns with adjustable topological charge and allows the acquisition of video data from the camera.

\section{RESULTS}

To characterize the beam, we verify its capacity to induce linear and orbital angular momentum on particles. Since the intensity of the beam follows a helico-conical profile, we can expect a dielectric particle approaching a spiral beam to be trapped along the region of highest photon density via gradient forces. The beam follows a spiral profile and so particles are expected to be trapped along the orbit and follow a spiral motion with an axial component of the momentum $\left(p_{z}\right)$ towards the direction of the beam propagation. Moreover, since the beam also has azimuthal component, we also expect that a tangential component of the momentum $\left(p_{\mathrm{T}}\right)$ is transferred to the particle.

To test the transfer of momentum on microscopic dielectric particles, Silica beads ( $2 \mu \mathrm{m}$ diameter) were dispersed in water and placed in a sample chamber made from microscope cover slips. The bead solution is bounded in a channel, which is around $100 \mu \mathrm{m}$ deep. The optical trapping portion of the experimental setup described in fig. 3 uses an inverted microscope (Leica). As such, the beams are directed upwards to allow beads settling at the bottom of the cover slip to be projected via the spiral beams. Figure 6 shows time lapse images of a particle trapped along the orbit as it approaches the spiral beam with $l=20$. The time interval between each frame is $40 \mathrm{~ms}$. With maximum photon density at the focus, 
majority of the momentum transfer occurs at this region sending the particle into a spiral motion. As $l$ is increased, the tangential momentum component $p_{\mathrm{T}}$ is increased following a reduction of the axial momentum component $p_{\mathrm{z}}$.
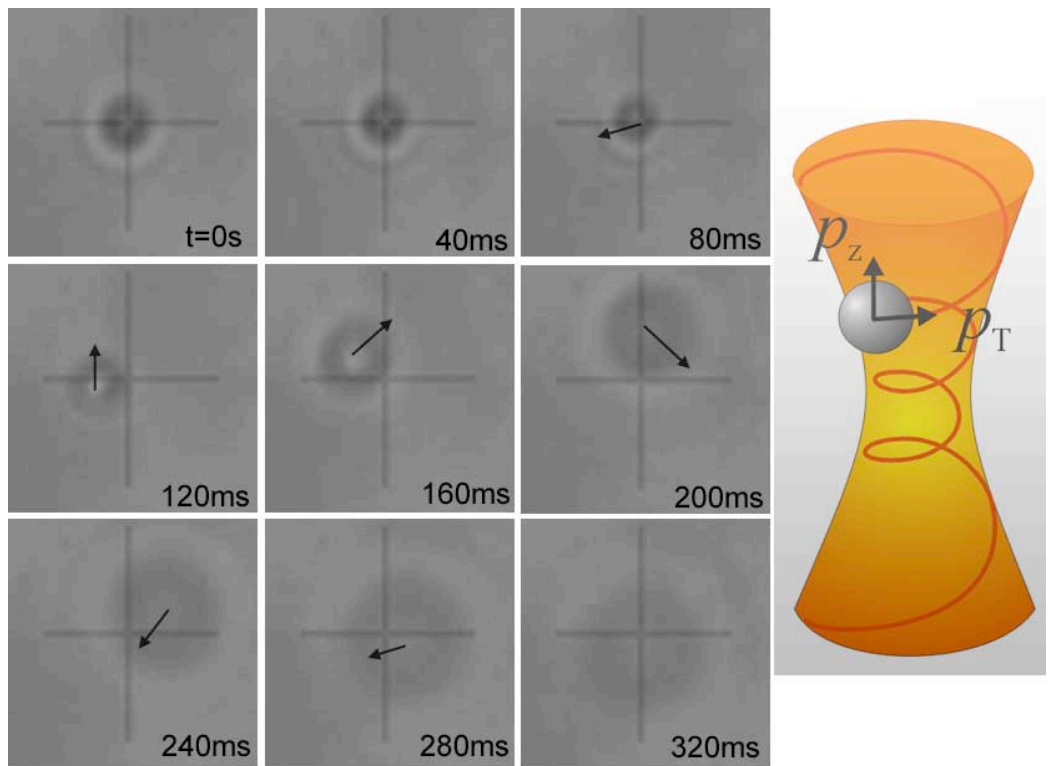

Figure 6. Time lapse images of a microbead trapped and guided along the orbit of an optical twister. Radiation pressure propels the particle towards the direction of the beam. However, the particle follows the spiral motion via the transfer of orbital angular momentum. Illustration on the right describes the motion of a particle in an optical twister.

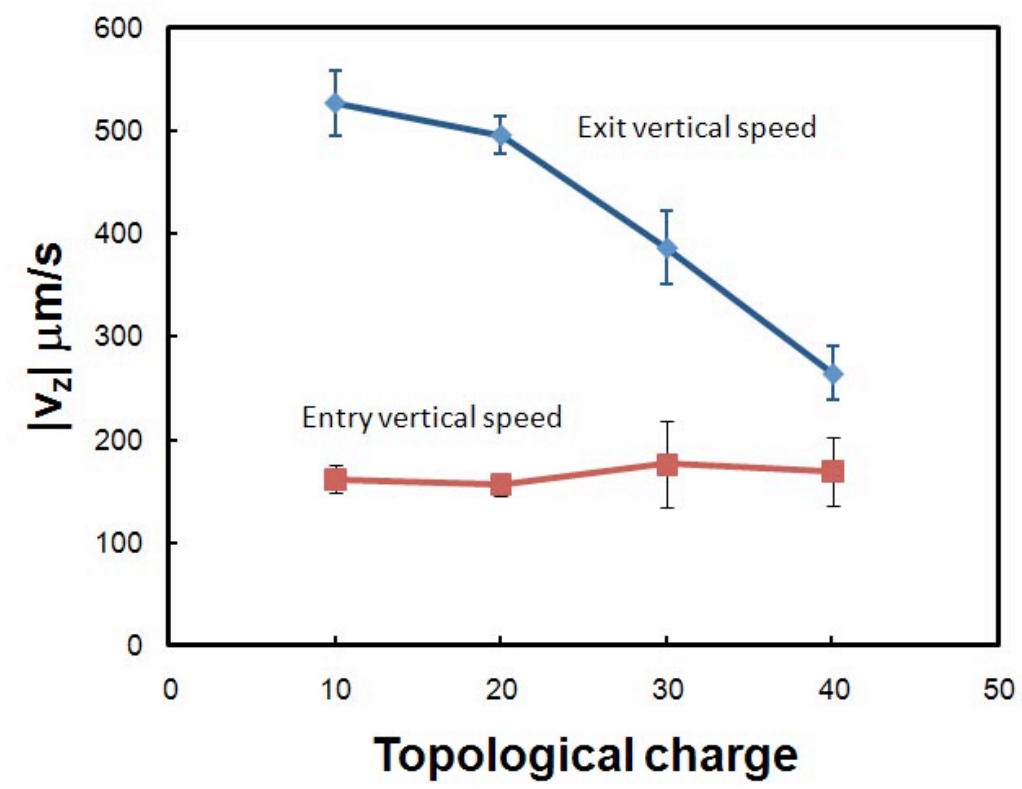

Figure 7. Vertical speeds of the microbeads as they enter $(\square)$ and exit $(\checkmark)$ an optical twister having different topological charges.

From the videos, we can track the particles to determine the motion of the particles as they enter and exit the optical twister. By taking note of the time at which the particles travel from the focus to a certain $z$-distance, we can analyze the 
momentum of the particle. Figure 7 shows the plot of the exit velocity, $v_{\mathrm{z}}=p_{\mathrm{z}} / m$, where $m$ is a constant that relates to the mass of the particle. As the topological charge, $l$, is increased the tangential component of the momentum is also increased. By conservation of momentum, a decrease in $v_{\mathrm{z}}$ is expected. The motion of the particles entering the optical twister does not seem to be affected by the increase in topological charge. When the particle is within the region of highest photon density, it undergoes effective transfer of linear and orbital angular momentum.

\section{CONCLUSIONS}

We have described a diffracting beam with a spiral profile on both the amplitude and phase of the beam. The three dimensional beam structure at the focal region shows an intensity distribution that can be accurately described as an "optical twister" as it propagates in the forward direction. In contrast to LG beams, optical twisters can have scalable orbital angular momentum but with minimal changes in radius. We show that spiral beams maintain a high concentration of photons at the focus even as the topological charge is increased. Finally, we have shown that an increasing orbital angular momentum induces more pronounced spiral motion on particles trapped along its orbit. Optical twisters can have direct application to fundamental studies of light and atoms such as in quantum entanglement of the OAM, toroidal traps for cold atoms and for optical manipulation of microscopic particles.

\section{ACKNOWLEDGEMENTS}

This collaborative work has been made possible via the Short Term Scientific Mission of the COST Action MP0604 and the Australian Academy of Science. VRDaria has been funded partially by the Australian National University - Office of the Vice-Chancellor. DPalima and JGlückstad acknowledge financial support from the Danish Technical Scientific Research Council (FTP). We also thank PJRodrigo for the discussions and helping us with the experiments.

\section{REFERENCES}

1. L. Allen, M.W. Beijersbergen, R.J.C. Spreeuw, J.P Woerdman, "Orbital angular momentum of light and the transformation of Laguerre-Gaussian laser modes," Phys rev. A 45, 8185-8189 (1992).

2. L. Allen, M. J. Padgett, and M. Babiker, "The orbital angular momentum of light," Prog. in Opt. 39, 291-372 (1999).

3. K.P. Marzlin, W. Zhang, E. Wright, "Vortex coupler for atomic Bose-Einstein condensates," Phys. Rev. Lett. 79, 4728-4731 (1997).

4. M. A. Clifford, J. Arlt, J. Courtial, K. Dholakia, "High-order Laguerre-Gaussian laser modes for studies of cold atoms," Opt. Commun. 156, 300-306 (1998).

5. J.E. Williams, M.J. Holland, "Preparing topological states of a Bose-Einstein condensate," Nature 401, 568-671 (1999).

6. M. F. Andersen, C. Ryu, P. Clade, V. Natarajan, A. Vaziri,K. Helmerson, W. D. Phillips, "Quantized rotation of atoms from photons with orbital angular momentum," Phys. Rev. Lett. 97, 170406 (2006).

7. H. He, M.E.J. Friese, N.R. Heckenberg, H. Rubinsztein-Dunlop, "Direct observation of transfer of angular momentum to absorptive particles from a laser beam with a phase singularity," Phys. Rev. Lett. 75, 826-829 (1995)

8. H. He, N. R. Heckenberg, and H. Rubinsztein-Dunlop, "Optical particle trapping with higher-order doughnut beams produced using high efficiency computer generated holograms,” J. Mod. Opt. 42, 217 (1995).

9. M.E.J. Friese, H. Rubinsztein-Dunlop, J. Enger, N.R. Heckenberg, “Optical angular momentum transfer to trapped absorbing particles," Phys. Rev. A 54, 1593-1596 (1996).

10. V. Garces-Chavez, K. Volke-Sepulveda, S. Chavez-Cerda, W. Sibbett, K. Dholakia, "Transfer of orbital angular momentum to an optically trapped low-index particle," Phys. Rev. A 66, 063402 (2002)

11. N. B. Simpson, K. Dholakia, L. Allen, M. J. Padgett, "The mechanical equivalence of the spin and orbital angular momentum of light: an optical spanner," Opt. Lett. 22, 52-54 (1997).

12. A. Mair, A. Vaziri, G. Weihs, A. Zeilinger, "Entanglement of the orbital angular momentum states of photon," Nature 412, 313-316 (2001) 
13. S. Franke-Arnold, S. M. Barnett, M. J. Padgett, M. J. Allen, "Two-photon entanglement of orbital angular momentum states," Phys. Rev. A 65, 033823 (2002).

14. C. A. Alonzo, P. J. Rodrigo, and J. Glückstad, "Helico-conical optical beams: a product of helical and conical phase fronts," Opt. Express 13, 1749-1760 (2005)'

15. E. R. Dufresne and D. G. Grier, "Optical tweezer arrays and optical substrates created with diffractive optical elements," Rev. Sci. Instrum. 69, 1974-1977 (1998).

16. M. Reicherter, T. Haist, E. U. Wagemann, and H. J. Tiziani, "Optical particle trapping with computer-generated holograms written on a liquid-crystal display," Opt. Lett. 24, 608-610 (1999).

17. J. Goodman, Introduction to Fourier Optics, (McGraw-Hill 1996). 